\title{
NITROGEN ASSIMILATING ENZYME OF AZOLLA AS INFLUENCED BY POTASSIC FERTILIZER
}

\section{S.Muruganayaki* and A.Jeyachitra**}

*Barathiyar University, Coimbatore,

** Madurai Kamarajar University, Madurai, India

\section{A R T I C L E I N F O}

\section{Article History:}

Received $14^{\text {th }}$ December, 2016

Received in revised form $16^{\text {th }}$ January, 2017

Accepted $8^{\text {th }}$ February, 2017

Published online $28^{\text {th }}$ March, 2017

\section{Key words:}

Azolla; Nitrogen fixation, Rice, Glutamine synthatese, Glutamate synthase and Glutamate dehydrogenase

\begin{abstract}
A B S T R A C T
Association between the Azolla and Anabena azollae resulted in fixation of atmospheric nitrogen through a process of reduction of atmospheric di-nitrogen mediated by the enzymes, glutamine synthetase (GS), glutamate synthase (GOGAT) and glutamate dehydrogenase (GDH) both under symbiotic and free-living conditions. The activity of these enzymes is increased by enrichment of nutrients especially $\mathrm{K}$ which usually applied to rice crop and improve the biological nitrogen fixation by Azolla. An incubation experiment was conducted by growing Azollae filiculoides with the agriculturally important potassic fertilizers (Potassium Chloride, Potassium shulphate) as main plot in seven concentrations $(0,5,10,20,30,40$ and $50 \mathrm{ppm}$ of $\mathrm{K})$ as sub-plots laid down in split-plot design replicated thrice to study the impact of these fertilizer on the activities of nitrogenfixing enzymes. The activity of glutamate synthase enzyme was predominant in azolla than other enzymes. The maximum activity of glutamate synthase $\left(1.567 \mathrm{n}\right.$ mole $\left.\min ^{-1} \mathrm{mg}^{-1}\right)$, glutamate dehydrogenase activity $\left(0.158 \mathrm{nmole} \min ^{-1} \mathrm{mg}^{-1}\right)$ and glutamine synthatase ( $0.908 \mathrm{n}$ mole $\left.\mathrm{min}^{-1} \mathrm{mg}^{-1}\right)$ was observed under the $40 \mathrm{ppm} \mathrm{K}$ solution especially on $30^{\text {th }}$ day after incubation. The potassium sulphate fertilizer was superior than the potassium chloride in influencing the growth and maintaining higher enzyme activity in azolla.
\end{abstract}

Copyright $₫ 2017$ S.Muruganayaki and A.Jeyachitra. This is an open access article distributed under the Creative Commons Attribution License, which permits unrestricted use, distribution, and reproduction in any medium, provided the original work is properly cited.

\section{INTRODUCTION}

Water fern Azolla fixes atmospheric nitrogen in association with nitrogen-fixing cynobacterium Anabbaena azollae and thereby serves as an effective biofertilizer in agriculture (Neirzwicki-Bauer, 1990 and Peters 1991). Azolla can be cultured in nutrient enriched water without significantly influencing their nitrogen fixation rates (Reddy, 1987). The response of Azolla to potassic fertilization is most effective in stimulating the growth of Azolla (Kannaiyan, 1990). A. pinnata - $A$. azollae association grew significantly better in the presence of nitrate as $\mathrm{KNO}_{3}$ (Rai and Rai, 2003). The ammonia assimilation is mediated by the enzyme glutamine synthatese (GS), glutamate synthase (GOGAT) and glutamate dehydrogenase (GDH) both under symbiotic and free-living conditions (Yates and Eady, 1989). The GDH appears to be involved in $\mathrm{NH}_{4}{ }^{+}$assimilation when the organisms grown at high $\mathrm{NH}_{4}^{+}$concentration but under N-limited conditions, the GS-GOGAT pathway predominate in $\mathrm{N}$ fixation. Further, the enrichment of nutrients especially $\mathrm{K}$ which is usually apllied to rice crop increases the activity of these enzymes and improve the biological nitrogen fixation by Azolla (Ray et.al, 1978).

*Corresponding author: S.Muruganayaki

Barathiyar University, Coimbatore,
Under these circumstances, a research was conducted to assess the enzymes responsible for $\mathrm{N}$ fixation under various concentrations of potassic fertilizers commonly used under Indian farming.

\section{MATERIALS AND METHODS}

An incubation experiment was conducted by growing Azollae filiculoides with 2 agriculturally important potassic fertilizers (Potassium Chloride, Potassium shulphate) as main plot in seven concentrations $(0,5,10,20,30,40$ and $50 \mathrm{ppm}$ of $\mathrm{K})$ as sub-plots laid down in split-plot design replicated thrice. The Azolla fern was grown in a tray with a dimension of $23 \times 15 \mathrm{x}$ $6 \mathrm{~cm}^{3}$ filled with 1.5 litres of potassic solutions. The fern was collected on $7^{\text {th }}, 15^{\text {th }}, 30^{\text {th }}, 60^{\text {th }}, 90^{\text {th }}$ and $120^{\text {th }}$ day after incubation / culturing, rinsed with distilled water and analysed for various biometric and biochemical parameters. The $\mathrm{N}$ assimilating enzyme such as Glutamate dehydrogenase, Glutamate synthase, and Glutamine synthatese were analysed by following standard methods as proposed by Dohertry (1970), Tempest et al. (1970) and Pateman (1969) respectively.

\section{Extraction of enzyme}

Nitrogen assimilating enzymes such as glutamate synthase and glutamate dehydrogenase were extracted from $1 \mathrm{~g}$ of azolla with $5 \mathrm{ml}$ of $100 \mathrm{mM}$ phosphate buffer $\mathrm{pH} 7.5$ containing $1 \mathrm{mM}$ disodium EDTA, 1mM dithioerythritol and 1 
$\%$ polyvinyl pyrrolidone (PVP) and centrifuged at 10,000rpm for 30 minutes at $4^{\circ} \mathrm{C}$.

\section{Glutamate synthase}

The enzyme extract $(0.2 \mathrm{ml})$ was added with $1 \mathrm{ml}$ of glutamine, $1 \mathrm{ml}$ of 2-oxoglutarate, $1 \mathrm{ml}$ of NADPH and 1.8 $\mathrm{ml}$ of Tris- $\mathrm{HCl}$ buffer $50 \mathrm{mM}$ at $\mathrm{pH} 7.6$, incubated for $15-30$ minutes and recorded absorbance at $340 \mathrm{~nm}$ in the Spectrophotometer.

\section{Glutamate dehydrogenase}

The enzyme extract $(0.2 \mathrm{ml})$ was added with $1 \mathrm{ml}$ of potassium phosphate buffer, $0.3 \mathrm{ml}$ of 2-oxoglutarate, $0.5 \mathrm{ml}$ of $\mathrm{NH}_{4} \mathrm{Cl}, 0.12 \mathrm{ml}$ of $\mathrm{NADH}$ or $\mathrm{NADPH}$ and $8 \mathrm{ml}$ of water, incubated for $15-30$ minutes at $37^{\circ} \mathrm{C}$ and recorded absorbance at $340 \mathrm{~nm}$ in the Spectrophotometer. The amount of NADH or NADPH oxidised per minute per mg of protein was calculated from the molar extinction co-efficient.

\section{Glutamine synthetase}

The enxyme was extracted from $1 \mathrm{~g}$ of azolla in $5 \mathrm{ml}$ of 50 $\mathrm{mM}$ Imidazole acetate buffer $\mathrm{pH} 7.8$ containing $0.5 \mathrm{mM}$ EDTA, $1 \mathrm{mM}$ dithioerythritol, $2 \mathrm{mM} \mathrm{MnCl}_{2}, 0.5 \%$ Glycerol and centrifuged at $10,000 \mathrm{rpm}$ for 30 minutes at $4^{\circ} \mathrm{C}$.

The enzyme extract $(0.2 \mathrm{ml})$ was added with $2 \mathrm{ml}$ of Lglutamine, $0.5 \mathrm{ml}$ of sodium arsenate, $0.3 \mathrm{ml}$ of $2 \mathrm{mM} \mathrm{MnCl}_{2}$, $0.5 \mathrm{ml}$ of hydroxylamine, $0.5 \mathrm{ml}$ of ADP and $2 \mathrm{ml}$ of $20 \mathrm{mM}$ Tris- $\mathrm{HCl}$ buffer, incubated for 30 minutes at $37^{\circ} \mathrm{C}$ and recorded absorbance at $340 \mathrm{~nm}$ in the Spectrophotometer after adding $1 \mathrm{ml}$ of ferric chloride.

\section{Statistical analysis}

The data were analysed statistically as per the procedures given by Panse and Sukhatme (1967) and the treatments were compared based on the critical difference for its effectiveness.

\section{Glutamate synthase (GS-GOGAT)}

The enzyme glutamate synthase dominates the nitrogen assimilation in Azolla than other enzymes. All the potassic fertilizers were effective in activating glutamate synthase and mean enzyme content of 0.788 and $0.957 \mathrm{n}$ mole $\mathrm{min}^{-1} \mathrm{mg}^{-1}$ was observed under potassium chloride and potassium sulphate respectively (Table 1a). Among the K fertilizer, the potassium sulphate was superior in maintaining higher enzyme content during all the days of incubation due to its sulphur bearing nature. The glutamate synthase activity was enhanced with the advancement of period of incubation upto $30^{\text {th }}$ day after incubation and decreased after that. The highest

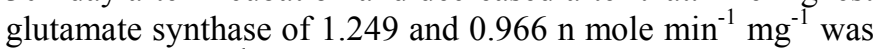
registered on $30^{\text {th }}$ day after incubation by potassium sulphate and potassium chloride respectively. The potassium sulphate fertilizer was superior in maintaining highest glutamate synthase content during all the days after incubation.

The potassium concentration also significantly influenced the glutamate synthase activity during all the days after incubation. In general, the content of glutamate synthase enzyme varied between 0.640 and $1.148 \mathrm{n}$ mole $\mathrm{min}^{-1} \mathrm{mg}^{-1}$ (Table 1a). The highest mean enzyme content of $1.148 \mathrm{n}$ mole $\mathrm{min}^{-1} \mathrm{mg}^{-1}$ was registered by the $40 \mathrm{ppm} \mathrm{K}$. As that of fertilizers, the glutamate synthase activity was enhanced with the advancement of period of incubation upto $30^{\text {th }}$ day after incubation and decreased thereafter. The maximum glutamate synthase content of $1.567 \mathrm{n}$ mole $\mathrm{min}^{-1} \mathrm{mg}^{-1}$ was recorded by the $40 \mathrm{ppm} \mathrm{K}$ solution on $30^{\text {th }}$ day after incubation followed by the same concentration on $60^{\text {th }}$ day after incubation $(1.264$ $\mathrm{n}$ mole $\mathrm{min}^{-1} \mathrm{mg}^{-1}$ ). The $40 \mathrm{ppm} \mathrm{K}$ registered higher glutamate synthase activity in all the days after incubation followed by $30 \mathrm{ppm} \mathrm{K}$ solution except on $15^{\text {th }}$ and $30^{\text {th }}$ day after incubation.

Table 1a Glutamate synthase enzyme (n mole $\mathrm{min}^{-1} \mathrm{mg}^{-1}$ ) in Azolla as influenced by the main effect of fertilizer and their concentration

\begin{tabular}{|c|c|c|c|c|c|c|c|}
\hline \multirow{2}{*}{ Treatment } & \multicolumn{6}{|c|}{ Period of incubation, Days } & \multirow{3}{*}{ Mean } \\
\hline & 7 & 15 & 30 & 60 & 90 & 120 & \\
\hline \multicolumn{7}{|c|}{ Fertilizer } & \\
\hline $\mathrm{F}_{1}(\mathrm{KCl})$ & 0.720 & 0.748 & 0.966 & 0.856 & 0.802 & 0.638 & 0.788 \\
\hline $\mathrm{F}_{2}\left(\mathrm{~K}_{2} \mathrm{SO}_{4}\right)$ & 0.823 & 1.009 & 1.249 & 1.002 & 0.889 & 0.757 & 0.957 \\
\hline Mean & 0.771 & 0.884 & 1.108 & 0.929 & 0.846 & 0.697 & \\
\hline $\mathrm{CD}(0.05)$ & 0.064 & 0.147 & 0.027 & 0.900 & 0.015 & 0.101 & \\
\hline \multicolumn{8}{|c|}{ Concentration } \\
\hline $\mathrm{C}_{1}(\mathbf{0} \mathrm{ppm})$ & 0.542 & 0.678 & 0.848 & 0.688 & 0.611 & 0.472 & 0.640 \\
\hline $\mathrm{C}_{2}(2 \mathrm{ppm})$ & 0.651 & 0.799 & 0.993 & 0.808 & 0.733 & 0.568 & 0.759 \\
\hline $\mathrm{C}_{3}(5 \mathrm{ppm})$ & 0.659 & 0.823 & 1.034 & 0.844 & 0.7757 & 0.563 & 0.780 \\
\hline $\mathrm{C}_{4}(10 \mathrm{ppm})$ & 0.755 & 0.868 & 0.973 & 0.859 & 0.825 & 0.686 & 0.828 \\
\hline $\mathrm{C}_{5}(20 \mathrm{ppm})$ & 0.839 & 0.898 & 1.155 & 0.979 & 0.906 & 0.772 & 0.925 \\
\hline $\mathrm{C}_{6}(30 \mathrm{ppm})$ & 0.907 & 0.930 & 1.140 & 0.999 & 0.965 & 0.848 & 0.973 \\
\hline $\mathrm{C}_{7}(40 \mathrm{ppm})$ & 0.990 & 1.086 & 1.567 & 1.264 & 1.077 & 0.904 & 1.148 \\
\hline $\mathrm{C}_{8}(50 \mathrm{ppm})$ & 0.830 & 0.947 & 1.149 & 0.991 & 0.893 & 0.767 & 0.928 \\
\hline Mean & 0.771 & 0.884 & 1.108 & 0.929 & 0.846 & 0.697 & \\
\hline $\mathrm{CD}(0.05)$ & 0.061 & 0.061 & 0.066 & 0.0611 & 0.072 & 0.069 & \\
\hline
\end{tabular}

\section{RESULTS AND DISCUSSIONS}

The ammonia assimilation is mediated by the enzyme glutamine synthatese (GS), glutamate synthase (GOGAT) and glutamate dehydrogenase (GDH) both under symbiotic and free-living conditions (Yates and Eady, 1989). The effect of K nutrient fertilizer and their concentration on the activities of these enzymes is presented and discussed below.
The interaction between the fertilizer and concentration was significant on $15^{\text {th }}, 30^{\text {th }}$ and $60^{\text {th }}$ day after incubation. Irrespective of the period of incubation the highest mean enzyme content of 1.019 and $1.278 \mathrm{n} \mathrm{mole} \mathrm{min}^{-1} \mathrm{mg}^{-1}$ was recorded when the azolla was grown under $40 \mathrm{ppm} \mathrm{K}$ solution as potassium chloride and potassium sulphate respectively (Table 1b). Irrespective of the concentration, the highest mean enzyme content of $1.108 \mathrm{n}$ mole $\mathrm{min}^{-1} \mathrm{mg}^{-1}$ was recorded on $30^{\text {th }}$ day after incubation. The highest enzyme content of 
1.267 and $1.868 \mathrm{n}$ mole $\mathrm{min}^{-1} \mathrm{mg}^{-1}$ was recorded when the azolla incubated under $40 \mathrm{ppm}$ of potassium chloride and potassium sulphate respectively which was 88 per cent and 82 per cent more than the control (0 ppm).

Table 1b Glutamate synthase enzyme (n mole $\mathrm{min}^{-1} \mathrm{mg}^{-1}$ ) in Azolla as influenced by the interaction effect of fertilizer and their concentration

\begin{tabular}{cccccccc}
\hline \multirow{2}{*}{ Treatment } & \multicolumn{7}{c}{ Period of incubation, Days } \\
\cline { 2 - 7 } & $\mathbf{7}$ & $\mathbf{1 5}$ & $\mathbf{3 0}$ & $\mathbf{6 0}$ & $\mathbf{9 0}$ & $\mathbf{1 2 0}$ & \\
\hline $\mathrm{F}_{1} \mathrm{C}_{1}$ & 0.477 & 0.526 & 0.671 & 0.599 & 0.542 & 0.412 & 0.538 \\
$\mathrm{~F}_{1} \mathrm{C}_{2}$ & 0.61 & 0.642 & 0.841 & 0.742 & 0.687 & 0.533 & 0.676 \\
$\mathrm{~F}_{1} \mathrm{C}_{3}$ & 0.581 & 0.653 & 0.906 & 0.78 & 0.689 & 0.472 & 0.680 \\
$\mathrm{~F}_{1} \mathrm{C}_{4}$ & 0.687 & 0.732 & 0.922 & 0.827 & 0.766 & 0.609 & 0.757 \\
$\mathrm{~F}_{1} \mathrm{C}_{5}$ & 0.804 & 0.779 & 1.066 & 0.923 & 0.891 & 0.716 & 0.863 \\
$\mathrm{~F}_{1} \mathrm{C}_{6}$ & 0.859 & 0.826 & 1.028 & 0.927 & 0.916 & 0.803 & 0.893 \\
$\mathrm{~F}_{1} \mathrm{C}_{7}$ & 0.934 & 0.939 & 1.267 & 1.103 & 1.045 & 0.823 & 1.019 \\
$\mathrm{~F}_{1} \mathrm{C}_{8}$ & 0.807 & 0.866 & 1.031 & 0.949 & 0.882 & 0.732 & 0.878 \\
$\mathrm{~F}_{2} \mathrm{C}_{1}$ & 0.606 & 0.831 & 1.025 & 0.778 & 0.681 & 0.531 & 0.742 \\
$\mathrm{~F}_{2} \mathrm{C}_{2}$ & 0.691 & 0.956 & 1.146 & 0.874 & 0.779 & 0.602 & 0.841 \\
$\mathrm{~F}_{2} \mathrm{C}_{3}$ & 0.738 & 0.994 & 1.163 & 0.908 & 0.824 & 0.653 & 0.880 \\
$\mathrm{~F}_{2} \mathrm{C}_{4}$ & 0.822 & 1.004 & 1.025 & 0.893 & 0.883 & 0.761 & 0.898 \\
$\mathrm{~F}_{2} \mathrm{C}_{5}$ & 0.874 & 1.018 & 1.244 & 1.035 & 0.922 & 0.826 & 0.987 \\
$\mathrm{~F}_{2} \mathrm{C}_{6}$ & 0.954 & 1.135 & 1.253 & 1.073 & 1.014 & 0.893 & 1.054 \\
$\mathrm{~F}_{2} \mathrm{C}_{7}$ & 1.046 & 1.234 & 1.868 & 1.426 & 1.109 & 0.983 & 1.278 \\
$\mathrm{~F}_{2} \mathrm{C}_{8}$ & 0.852 & 1.009 & 1.267 & 1.034 & 0.905 & 0.801 & 0.978 \\
$\mathrm{Mean}_{2}$ & 0.771 & 0.884 & 1.108 & 0.929 & 0.846 & 0.697 & \\
$\mathrm{CD}(0.05)$ & & & & & & & \\
$\mathrm{F}_{\text {at }} \mathrm{C}$ & $\mathrm{NS}$ & 0.157 & 0.090 & 0.113 & $\mathrm{NS}$ & $\mathrm{NS}$ & \\
$\mathrm{C}_{\text {at }} \mathrm{F}$ & $\mathrm{NS}$ & 0.085 & 0.093 & 0.086 & $\mathrm{NS}$ & $\mathrm{NS}$ & \\
\hline
\end{tabular}

The observed higher activity of glutamate synthase enzyme than other enzymes was due to its active involvement in GSGOGAT pathway to incorporate nitrogen in the amide position of glutamine and transferred to $\alpha$ - position of $\alpha-$ keto glutamate in the host azolla as outlined by Boussiba and Gibson (1991). The suppressed enzyme content at the higher concentration was mainly due to its toxicity as observed by Venkataraman and Kannaiyan (1986).

\section{Glutamate dyhydrogenase (GDH)}

The enzyme glutamate dehydrogenase content was increased with the advancement of period of incubation in both fertilizers and their concentrations up to 30 days of incubation. In general, the potassium sulphate registered highest mean enzyme content of $0.095 \mathrm{n}$ mole $\mathrm{min}^{-1} \mathrm{mg}^{-1}$ (Table $2 \mathrm{a})$ followed by the potassium chloride $(0.073 \mathrm{n}$ mole $\left.\min ^{-1} \mathrm{mg}^{-1}\right)$.
The potassium sulphate significantly maintained higher glutamate dehydrogenase content during all the days of incubation and highest content of $0.130 \mathrm{n}$ mole $\min ^{-1} \mathrm{mg}^{-1}$ was observed on $30^{\text {th }}$ day after incubation. The potassium chloride fertilizer also produced highest glutamate dehydrogenase content of $0.116 \mathrm{n} \mathrm{mole} \mathrm{min}^{-1} \mathrm{mg}^{-1}$ on the same $30^{\text {th }}$ day of incubation.

The enzyme content increased with the advancement of period of incubation when the azolla was incubated with various $\mathrm{K}$ concentrated solution. In general, the highest mean glutamate dehydrogenase enzyme content of $0.107 \mathrm{n}$ mole $\mathrm{min}^{-1} \mathrm{mg}^{-1}$ was registered by $40 \mathrm{ppm}$ of $\mathrm{K}$ solution followed

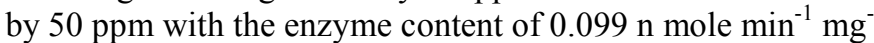
1 irrespective of period of incubation (Table 2a). The significantly highest glutamate dehydrogenase activity $(0.158$ $\mathrm{n}$ mole $\mathrm{min}^{-1} \mathrm{mg}^{-1}$ ) was noticed on $30^{\text {th }}$ day after incubation under $40 \mathrm{ppm} \mathrm{K}$ followed by $50 \mathrm{ppm}$ in all the days of incubation except $15^{\text {th }}$ and $30^{\text {th }}$ days after incubation in which the $30 \mathrm{ppm} \mathrm{K}$ registered next highest enzyme content. It indicates the higher concentration of $\mathrm{K}$ restrict the azolla growth by its toxicity. Though the $40 \mathrm{ppm} \mathrm{K}$ significantly maintained highest enzyme content during all the days of incubation, which was on par with the next lowest (30 ppm) and next highest $(50 \mathrm{ppm})$ concentration on $15^{\text {th }}$ day after incubation. This shows the insufficient and excess $\mathrm{K}$ requirement for azolla growth during these days of incubation.

Interaction between the potassic fertilizer and their concentration significantly influenced glutamate dehydrogeanse enzyme activity during $15^{\text {th }}$ and $30^{\text {th }}$ days after incubation (Table 2b). The average glutamate dehydrogenase content varied between 0.046 and $0.119 \mathrm{n} \mathrm{mole} \mathrm{min}^{-1} \mathrm{mg}^{-1}$. The highest enzyme content of $0.163 \mathrm{n}$ mole $\mathrm{min}^{-1} \mathrm{mg}^{-1}$ was maintained by the application potassium sulphate as $40 \mathrm{ppm}$ followed by $30 \mathrm{ppm}\left(0.155 \mathrm{n}\right.$ mole $\left.\mathrm{min}^{-1} \mathrm{mg}^{-1}\right)$ which were on par with each other. The same trend was observed during $15^{\text {th }}$ day after incubation. The synthesis of more glutamate dehydrogenase enzyme by the potassium sulphate was mainly due to high water soluble nature of the fertilizer and bearing of S which facilitates higher growth of azolla. Meeks et al. (1985) have reported the same findings. Tilo et al. (1989) concluded that potassium dihydrogen phosphate and potassium sulphate were equally effective in increasing the biomass production of Azolla.

Table 2a Glutamate dehydrogenase enzyme (n mole $\mathrm{min}^{-1} \mathrm{mg}^{-1}$ ) in Azolla as influenced by the main effect of fertilizer and their concentration

\begin{tabular}{|c|c|c|c|c|c|c|c|}
\hline \multirow{2}{*}{ Treatment } & \multicolumn{6}{|c|}{ Period of incubation, Days } & \multirow{2}{*}{ Mean } \\
\hline & 7 & 15 & 30 & 60 & 90 & 120 & \\
\hline \multicolumn{8}{|c|}{ Fertilizer } \\
\hline $\mathrm{F}_{1}(\mathrm{KCl})$ & 0.038 & 0.102 & 0.116 & 0.077 & 0.057 & 0.047 & 0.073 \\
\hline $\mathrm{F}_{2}\left(\mathrm{~K}_{2} \mathrm{SO}_{4}\right)$ & 0.068 & 0.114 & 0.130 & 0.100 & 0.084 & 0.076 & 0.095 \\
\hline Mean & 0.053 & 0.108 & 0.123 & 0.088 & 0.071 & 0.062 & \\
\hline $\mathrm{CD}(0.05)$ & 0.002 & 0.009 & 0.004 & 0.008 & 0.002 & 0.004 & \\
\hline \multicolumn{8}{|c|}{ Concentration } \\
\hline $\mathrm{C}_{1}(0 \mathrm{ppm})$ & 0.040 & 0.070 & 0.077 & 0.059 & 0.050 & 0.045 & 0.057 \\
\hline $\mathrm{C}_{2}(2 \mathrm{ppm})$ & 0.046 & 0.078 & 0.097 & 0.072 & 0.059 & 0.053 & 0.067 \\
\hline $\mathrm{C}_{3}(5 \mathrm{ppm})$ & 0.049 & 0.100 & 0.099 & 0.075 & 0.062 & 0.055 & 0.073 \\
\hline $\mathrm{C}_{4}(10 \mathrm{ppm})$ & 0.052 & 0.114 & 0.117 & 0.085 & 0.068 & 0.060 & 0.082 \\
\hline $\mathrm{C}_{5}(20 \mathrm{ppm})$ & 0.054 & 0.113 & 0.134 & 0.094 & 0.074 & 0.064 & 0.089 \\
\hline $\mathrm{C}_{6}(30 \mathrm{ppm})$ & 0.055 & 0.130 & 0.151 & 0.104 & 0.079 & 0.067 & 0.098 \\
\hline $\mathrm{C}_{7}(40 \mathrm{ppm})$ & 0.069 & 0.134 & 0.158 & 0.113 & 0.091 & 0.080 & 0.107 \\
\hline $\mathrm{C}_{8}(50 \mathrm{ppm})$ & 0.060 & 0.125 & 0.150 & 0.105 & 0.083 & 0.071 & 0.099 \\
\hline Mean & 0.053 & 0.108 & 0.123 & 0.088 & 0.071 & 0.062 & \\
\hline $\mathrm{CD}(0.05)$ & 0.003 & 0.008 & 0.005 & 0.007 & 0.004 & 0.003 & \\
\hline
\end{tabular}


Table 2 b Glutamate dehydrogenase enzyme (n mole min $^{-1}$ $\mathrm{mg}^{-1}$ ) in Azolla as influenced by the interaction effect of fertilizer and their concentration

\begin{tabular}{|c|c|c|c|c|c|c|c|}
\hline \multirow{2}{*}{ Treatment } & \multicolumn{6}{|c|}{ Period of incubation, Days } & \multirow{2}{*}{ Mean } \\
\hline & 7 & 15 & 30 & 60 & 90 & 120 & \\
\hline $\mathrm{F}_{1} \mathrm{C}_{1}$ & 0.024 & 0.068 & 0.071 & 0.048 & 0.036 & 0.030 & 0.046 \\
\hline $\mathrm{F}_{1} \mathrm{C}_{2}$ & 0.030 & 0.075 & 0.084 & 0.057 & 0.044 & 0.037 & 0.055 \\
\hline $\mathrm{F}_{1} \mathrm{C}_{3}$ & 0.035 & 0.085 & 0.084 & 0.060 & 0.047 & 0.041 & 0.059 \\
\hline $\mathrm{F}_{1} \mathrm{C}_{4}$ & 0.038 & 0.111 & 0.109 & 0.074 & 0.056 & 0.047 & 0.073 \\
\hline $\mathrm{F}_{1} \mathrm{C}_{5}$ & 0.039 & 0.108 & 0.129 & 0.084 & 0.062 & 0.050 & 0.079 \\
\hline $\mathrm{F}_{1} \mathrm{C}_{6}$ & 0.040 & 0.122 & 0.147 & 0.094 & 0.067 & 0.053 & 0.087 \\
\hline $\mathrm{F}_{1} \mathrm{C}_{7}$ & 0.052 & 0.126 & 0.152 & 0.102 & 0.077 & 0.065 & 0.096 \\
\hline $\mathrm{F}_{1} \mathrm{C}_{8}$ & 0.042 & 0.120 & 0.148 & 0.095 & 0.069 & 0.055 & 0.088 \\
\hline $\mathrm{F}_{2} \mathrm{C}_{1}$ & 0.056 & 0.071 & 0.083 & 0.070 & 0.063 & 0.059 & 0.067 \\
\hline $\mathrm{F}_{2} \mathrm{C}_{2}$ & 0.062 & 0.081 & 0.109 & 0.086 & 0.074 & 0.068 & 0.080 \\
\hline $\mathrm{F}_{2} \mathrm{C}_{3}$ & 0.063 & 0.115 & 0.114 & 0.089 & 0.076 & 0.069 & 0.088 \\
\hline $\mathrm{F}_{2} \mathrm{C}_{4}$ & 0.065 & 0.116 & 0.124 & 0.095 & 0.080 & 0.072 & 0.092 \\
\hline $\mathrm{F}_{2} \mathrm{C}_{5}$ & 0.068 & 0.117 & 0.139 & 0.104 & 0.086 & 0.077 & 0.099 \\
\hline $\mathrm{F}_{2} \mathrm{C}_{6}$ & 0.070 & 0.137 & 0.155 & 0.113 & 0.091 & 0.081 & 0.108 \\
\hline $\mathrm{F}_{2} \mathrm{C}_{7}$ & 0.085 & 0.142 & 0.163 & 0.124 & 0.105 & 0.095 & 0.119 \\
\hline $\mathrm{F}_{2} \mathrm{C}_{8}$ & 0.077 & 0.130 & 0.152 & 0.115 & 0.096 & 0.086 & 0.109 \\
\hline Mean & 0.053 & 0.108 & 0.123 & 0.088 & 0.071 & 0.062 & \\
\hline \multicolumn{8}{|l|}{$\mathrm{CD}(0.05)$} \\
\hline $\mathrm{F}$ at $\mathrm{C}$ & NS & 0.013 & 0.008 & NS & NS & NS & \\
\hline $\mathrm{C}$ at $\mathrm{F}$ & NS & 0.011 & 0.007 & NS & NS & NS & \\
\hline
\end{tabular}

\section{Glutamine synthetase}

The main factor of potassic fertilizer and its concentration significantly influenced the glutamine synthatese content during all the days of incubation except $7^{\text {th }}$ and $30^{\text {th }}$ day. In general, the fertilizer potassium sulphate registered highest mean glutamine synthatese of $0.731 \mathrm{n}$ mole $\mathrm{min}^{-1} \mathrm{mg}^{-1}$ (Table

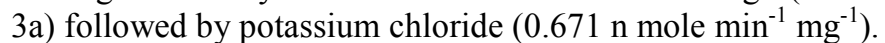
The enzyme activity was more during initial stage of incubation and maintained almost constant value during the later stages of incubation.
Generally, the mean glutamine synthatese content varied between 0.586 and $0.865 \mathrm{n}$ mole $\mathrm{min}^{-1} \mathrm{mg}^{-1}$ under the interaction effect of fertilizer with its concentration (Table 3b).

Table 3b Glutamine synthatase enzyme (n mole $\mathrm{min}^{-1} \mathrm{mg}^{-1}$ ) in Azolla as influenced by the interaction effect of fertilizer and their concentration

\begin{tabular}{|c|c|c|c|c|c|c|c|}
\hline \multirow{2}{*}{ Treatment } & \multicolumn{6}{|c|}{ Period of incubation, Days } & \multirow{2}{*}{ Mean } \\
\hline & 7 & 15 & 30 & 60 & 90 & 120 & \\
\hline $\mathrm{F}_{1} \mathrm{C}_{1}$ & 0.504 & 0.581 & 0.726 & 0.615 & 0.560 & 0.532 & 0.586 \\
\hline $\mathrm{F}_{1} \mathrm{C}_{2}$ & 0.516 & 0.588 & 0.758 & 0.637 & 0.577 & 0.546 & 0.604 \\
\hline $\mathrm{F}_{1} \mathrm{C}_{3}$ & 0.536 & 0.640 & 0.769 & 0.653 & 0.594 & 0.565 & 0.626 \\
\hline $\mathrm{F}_{1} \mathrm{C}_{4}$ & 0.552 & 0.672 & 0.806 & 0.679 & 0.616 & 0.584 & 0.652 \\
\hline $\mathrm{F}_{1} \mathrm{C}_{5}$ & 0.595 & 0.677 & 0.826 & 0.711 & 0.653 & 0.624 & 0.681 \\
\hline $\mathrm{F}_{1} \mathrm{C}_{6}$ & 0.670 & 0.701 & 0.833 & 0.752 & 0.711 & 0.690 & 0.726 \\
\hline $\mathrm{F}_{1} \mathrm{C}_{7}$ & 0.742 & 0.742 & 0.880 & 0.811 & 0.777 & 0.759 & 0.785 \\
\hline $\mathrm{F}_{1} \mathrm{C}_{8}$ & 0.650 & 0.680 & 0.825 & 0.738 & 0.694 & 0.672 & 0.710 \\
\hline $\mathrm{F}_{2} \mathrm{C}_{1}$ & 0.509 & 0.613 & 0.723 & 0.616 & 0.563 & 0.536 & 0.593 \\
\hline $\mathrm{F}_{2} \mathrm{C}_{2}$ & 0.570 & 0.691 & 0.789 & 0.680 & 0.625 & 0.597 & 0.659 \\
\hline $\mathrm{F}_{2} \mathrm{C}_{3}$ & 0.592 & 0.804 & 0.821 & 0.707 & 0.649 & 0.621 & 0.699 \\
\hline $\mathrm{F}_{2} \mathrm{C}_{4}$ & 0.598 & 0.842 & 0.862 & 0.730 & 0.664 & 0.631 & 0.721 \\
\hline $\mathrm{F}_{2} \mathrm{C}_{5}$ & 0.614 & 0.853 & 0.879 & 0.747 & 0.680 & 0.647 & 0.737 \\
\hline $\mathrm{F}_{2} \mathrm{C}_{6}$ & 0.725 & 0.863 & 0.906 & 0.816 & 0.770 & 0.748 & 0.805 \\
\hline $\mathrm{F}_{2} \mathrm{C}_{7}$ & 0.812 & 0.898 & 0.936 & 0.874 & 0.843 & 0.828 & 0.865 \\
\hline $\mathrm{F}_{2} \mathrm{C}_{8}$ & 0.703 & 0.843 & 0.850 & 0.777 & 0.740 & 0.721 & 0.772 \\
\hline Mean & 0.618 & 0.731 & 0.824 & 0.721 & 0.670 & 0.644 & \\
\hline \multicolumn{8}{|l|}{$\mathrm{CD}(0.05)$} \\
\hline $\mathrm{F}$ at $\mathrm{C}$ & NS & 0.054 & NS & NS & 0.025 & NS & \\
\hline $\mathrm{C}$ at $\mathrm{F}$ & NS & 0.029 & NS & NS & 0.026 & NS & \\
\hline
\end{tabular}

Though the significant interaction between the potassic fertilizer and concentrations in maintaining glutamine synthatese content was observed during $15^{\text {th }}$ and $90^{\text {th }}$ day after incubation, the highest glutamine synthatese content of 0.936 $\mathrm{n}$ mole $\mathrm{min}^{-1} \mathrm{mg}^{-1}$ was recorded by the $40 \mathrm{ppm}$ of potassium sulphate on $30^{\text {th }}$ days after incubation.

Table 3a. Glutamine synthatase enzyme $\left(\mathrm{n}\right.$ mole $\left.\mathrm{min}^{-1} \mathrm{mg}^{-1}\right)$ in Azolla as influenced by the main effect of fertilizer and their concentration

\begin{tabular}{|c|c|c|c|c|c|c|c|}
\hline \multirow{2}{*}{ Treatment } & \multicolumn{6}{|c|}{ Period of incubation, Days } & \multirow{2}{*}{ Mean } \\
\hline & \multicolumn{6}{|c|}{ Fertilizer } & \\
\hline $\mathrm{F}_{1}(\mathrm{KCl})$ & 0.596 & 0.660 & 0.803 & 0.700 & 0.648 & 0.622 & 0.671 \\
\hline $\mathrm{F}_{2}\left(\mathrm{~K}_{2} \mathrm{SO}_{4}\right)$ & 0.640 & 0.801 & 0.846 & 0.743 & 0.692 & 0.666 & 0.731 \\
\hline Mean & 0.618 & 0.731 & 0.824 & 0.721 & 0.670 & 0.644 & \\
\hline $\mathrm{CD}(0.05)$ & NS & 0.051 & NS & 0.031 & 0.005 & 0.038 & \\
\hline \multicolumn{8}{|c|}{ Concentration } \\
\hline $\mathrm{C}_{1}(0 \mathrm{ppm})$ & 0.507 & 0.597 & 0.725 & 0.616 & 0.562 & 0.534 & 0.590 \\
\hline $\mathrm{C}_{2}(2 \mathrm{ppm})$ & 0.543 & 0.640 & 0.774 & 0.659 & 0.601 & 0.572 & 0.631 \\
\hline $\mathrm{C}_{3}(5 \mathrm{ppm})$ & 0.564 & 0.722 & 0.795 & 0.680 & 0.622 & 0.593 & 0.663 \\
\hline $\mathrm{C}_{4}(10 \mathrm{ppm})$ & 0.575 & 0.757 & 0.834 & 0.705 & 0.640 & 0.608 & 0.686 \\
\hline $\mathrm{C}_{5}(20 \mathrm{ppm})$ & 0.605 & 0.765 & 0.853 & 0.729 & 0.667 & 0.636 & 0.709 \\
\hline $\mathrm{C}_{6}(30 \mathrm{ppm})$ & 0.698 & 0.782 & 0.870 & 0.784 & 0.741 & 0.719 & 0.765 \\
\hline $\mathrm{C}_{7}(40 \mathrm{ppm})$ & 0.777 & 0.820 & 0.908 & 0.843 & 0.810 & 0.794 & 0.825 \\
\hline $\mathrm{C}_{8}(50 \mathrm{ppm})$ & 0.677 & 0.762 & 0.838 & 0.758 & 0.717 & 0.697 & 0.741 \\
\hline Mean & 0.618 & 0.731 & 0.824 & 0.721 & 0.670 & 0.644 & \\
\hline $\mathrm{CD}(0.05)$ & 0.110 & 0.021 & 0.073 & 0.029 & 0.018 & 0.027 & \\
\hline
\end{tabular}

Irrespective of fertilizers used, all the $\mathrm{K}$ concentrated solution significantly influenced the glutamine synthatase content during all the days of incubation. The mean enzyme content varied between 0.590 and $0.825 \mathrm{n}$ mole $\mathrm{min}^{-1} \mathrm{mg}^{-1}$ (Table 3a). Incubation of azolla with $40 \mathrm{ppm} \mathrm{K}$ exhibited more glutamine synthatase content during all the days but it was on par with 30 and $50 \mathrm{ppm}$ solution during early stages of incubation. Though the maximum glutamine synthatase content of 0.908 $\mathrm{n}$ mole $\mathrm{min}^{-1} \mathrm{mg}^{-1}$ was observed under $40 \mathrm{ppm}$ potassic solution which were on par with the 20,30 and 50 ppm on $30^{\text {th }}$ day after incubation
The same concentration significantly maintained highest glutamine synthatase content of 0.898 and $0.873 \mathrm{n}$ mole $\min ^{-1}$ $\mathrm{mg}^{-1}$ on $15^{\text {th }}$ and $90^{\text {th }}$ days after incubation respectively.

Both $\mathrm{K}$ fertilizers were effective in synthesising the glutamine synthatese enzyme and a typical increase was noticed in potassium sulphate. The $40 \mathrm{ppm} \mathrm{K}$ was sufficient for synthesising adequate amount of glutamine synthatese (Pan and Coote,1979). Paters et al. (1979) confirmed the host assimilation of nutrients with respect of GS activity and Uheda (1986) observed the higher GS and GDH activity in cavity hairs than leaves in Azolla. 


\section{CONCLUSIONS}

The activity of glutamate synthase enzyme was predominant in azolla than other enzymes due to an active involvement of GS-GOGAT pathway to incorporate nitrogen in the amide position of glutamine. The maximum activity of glutamate synthase (1.567 n mole $\left.\mathrm{min}^{-1} \mathrm{mg}^{-1}\right)$, glutamate dehydrogenase activity $\left(0.158 \mathrm{n}\right.$ mole $\left.\mathrm{min}^{-1} \mathrm{mg}^{-1}\right)$ and glutamine synthatase

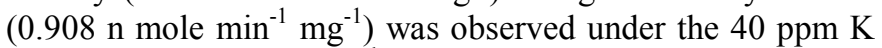
solution especially on $30^{\text {th }}$ day after incubation. The activity of the enzyme at $40 \mathrm{ppm}$ was on par with 30 and $50 \mathrm{ppm}$ solution during early stages of incubation which implies the insufficient and toxicity of $\mathrm{K}$ respectively for azolla. The potassium sulphate fertilizer was superior than the potassium chloride in influencing the growth and maintaining higher enzyme activity in azolla.

\section{Reference}

Boussiba, S and Gibson J. 1991. Ammonia translocation in cyanobacteria. FEMS Microbial. Rev., 88: 1- 14.

Dohertry, D. 1970. L-glutamate dehydrogenase, In Methods in Enzymology, (eds.) Tabor H., Tabor, C.W. Academic Press, London.

Meeks,J.C., N. Steinberg, CM. Joseph, C.S. Enderlin, P.A. Jorgensen, G.A,. Peters.1985. Assimilation of exogenous and dinitrogen-derived ${ }^{13} \mathrm{NH}_{4}$ by Anabaena azollae separated from Azolla caroliniana Wild, Arch.Microbiol.142:229-233

Kannaiyan, S. 1990. Biotechnology of Biofertilizer for Rice Crop. Tamil Nadu Agric. Univ., Coimbatore, Tamil Nadu, India.

Neirzwicki-Bauer, S. A .1990. Azolla-Anabaena symbiosis: Use in Agriculture: In Hand book of symbiotic cyanobacteria (ed.) A.N.Rai, CRC Press, Boca Raton, Florida, USA. Pp. 119-136.
Pan, F. L., and J. G. Coote. 1979. Glutamine synthetase and glutamate synthetase activities during growth and sporulation in Bacillus subtilis. J. Gen. Microbiol. 112:373-377.

Panse, V.G. and Sukhatme, P.V.1985. Statistical Methods for Agricultural Workers. $4^{\text {th }}$ edn. Indian Council of Agricultural Research, New Delhi. P.347.

Pateman, J.A. 1969. Biochem. J. 115: 769.

Peters, G. A. 1991. Azolla and other plant cyanobacteria symbiosis: Aspects of form and function. Pl. Soil. 137: 25-36

Rai, A.K.and Rai, V.2003. Effect of $\mathrm{NaCl}$ on growth, nitrate uptake and reduction and nitrogenase activity of Azolla pinnata Anabaena azollae. Plant Science 164: 61-69.

Ray, T.B., Peters, G.A., Toia, R.E., Jr Mayne, B.C. 1978. Azolla-Anabaena relationship. VII Distribution of ammonia assimilating enzymes, protein, and chlorophyll between host and symbiont. Pl. Physiol. 62: 463 - 467.

Reddy, K.R. 1987. Nitrogen fixation by Azolla cultured in nutrient enriched water. J. Aquat. Plant Manage. 25: 43-48.

Tempset D.W., Meers, J.C and Brow, C.M. 1970. Biochem.j.177:405

Tilo, S.N., Baniao, R.D., SanValentia, G.O. and Lales, J.S. 1989. Soil fertility assessment and fertilizer use on Azolla. In: Azolla. Its culture, management and utilization in the Phillipines. National Azolla Action Programme, UPLB, Los Banos, Phillipines. Pp. 139 -166.

Uheda, E. 1986. Isolation of empty packets from Anabaena free Azolla. Plant Cell Physiol. 27: 1187 - 1190.

Venkataraman, S and Kannaiyan, S. 1986. Effect of fertilizer nitrogen, neem cake and herbicide-machete on the ammonia assimilating enzymes of Azolla. Egypt J. Microbiol. 21: 163 - 170.

Yates, M.G and Eady, R.R. 1989. The physiology and regulation of nitrogen fixation. In: Recent Advances in Biological nitrogen fixation (ed.) N.S.Subba Rao, Oxford IBH Publishing Co., New Delhi. India. Pp. 88-120.

\section{Please cite this article in press as:}

S. Muruganayaki and A. Jeyachitra (2017) Nitrogen assimilating enzyme of azolla as influenced by potassic fertilizer, International Journal of Current Advanced Research, 6(03), pp. 3012-3016. http://dx.doi.org/10.24327/ijcar.2017. 3016.0165 